\title{
Heilmittelregresse vermeiden dank Verordnung per Software
}

\begin{abstract}
Wer zur Verordnung von Heilmitteln sein Praxisverwaltungssystem (PVS) nutzt, darf ab Januar 2017 nur noch zer-
\end{abstract}

tifizierte Software verwenden. Damit soll sichergestellt werden, dass alle verwendeten Softwareprodukte die Informationen enthalten, die Ärzte für eine korrekte Verordnung benötigen. Die KBV wurde mit der Zertifizierung beauftragt.

\section{MMW-KOMMENTAR}

Die neue Verordnungssoftware enthält alle Informationen der Heilmittelrichtlinie inklusive des Heilmittelkatalogs, und zwar immer auf dem aktuellen Stand. Sie prüft die Plausibilität der eingegebenen Verordnungsdaten und gibt dem Arzt Hinweise, wenn etwas nicht in Ordnung ist - z. B. wenn das Formular unvollständig ausgefüllt ist, die eingegebene Verordnungsmen-

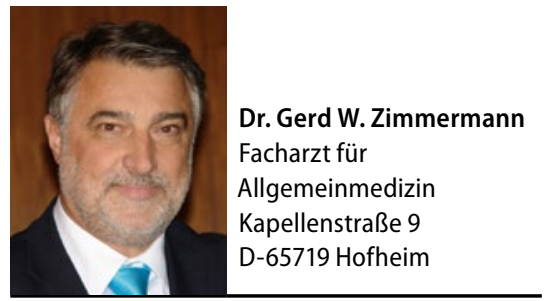

ge zu hoch ist oder bei einer Verordnung außerhalb des Regelfalls die medizinische Begründung fehlt.

Dem Arzt wird außerdem verbindlich angezeigt, wenn seine Eingaben einen besonderen Verordnungsbedarf (Praxisbesonderheiten) oder einen langfristigen Heilmittelbedarf begründen. Dafür werden die ICD-10Codes mit dem Indikationsschlüssel und ggf. noch mit dem Patientenalter oder dem Verordnungszeitraum abgeglichen. Das ist ein guter Service, denn ein besonderer Verordnungsbedarf und ein langfristiger Heilmittelbedarf bedeuten, dass die Verordnungen bei einer etwaigen Wirtschaftlichkeitsprüfung nicht berücksichtigt werden. Die Arztpraxis wird somit vor eventuellen Regressforderungen geschützt.

Eine gute Nachricht auch für Papierfreunde: Wer Vordrucke per Hand ausfüllt, weil er nur sehr wenige Heilmittelverordnungen ausstellt, kann dies auch weiterhin tun.

\section{Qualitätsmanagement in der Praxis neu geregelt}

\begin{abstract}
— Vertragsärzte und Vertragspsychotherapeuten sind seit 2004 durch den $\$ 135$ a SGB V verpflichtet, ein einrichtungsinternes Qualitätsmanagement (QM) einzuführen und weiterzuentwickeln. In der QM-Richtlinie des Gemeinsamen Bundesausschusses (G-BA) sind dafür Ziele, Grundsätze, Instrumente und Zeitrahmen festgelegt. Helfen können strukturierte Leitfäden, an denen sich die Praxen orientieren können, sogenannte QM-Verfahren. Bekannt sind u. a. "Qualität und Entwicklung in Praxen" (QEP), KTQ oder EPA. Verpflichtend sind sie nicht.
\end{abstract}

\section{MMW-KOMMENTAR}

Ab sofort gelten für Praxen und Krankenhäuser einheitliche QM-Anforderungen. Dafür ist am 16. November 2016 eine neue G-BARichtlinie in Kraft getreten. Sie löst die drei bisherigen Richtlinien für den vertragsärztlichen, den vertragszahnärztlichen und den stationären Bereich ab.

In der Richtlinie werden verschiedene Anwendungsbereiche wie Notfallmanagement und Hygienemanagement aufgeführt. Neu aufgenommen wurden Arzneimitteltherapiesicherheit, Schmerzmanagement und Maßnahmen zur Vermeidung von Stürzen. Neben regelmäßigen Patientenbefragungen sollen zukünftig auch Praxismitarbeitermöglichst anonym - befragt werden, damit die Praxisleitung Anregungen für Veränderungen und Verbesserungen erhält. Da die Ergebnisse der bisherigen Erhebungen zum Stand der Umsetzung und der Weiterentwicklung des QM stabil sind, finden die Stichprobenprüfungen gemäß einer Übergangsregelung nicht mehr jährlich, sondern nur noch alle zwei Jahre statt - die nächste 2017. Entsprechen die Ergebnisse nicht den Anforderungen der QM-Richtlinie, werden die Praxen von den QM-Kommissionen der KVen beraten. Sanktionen gibt es auch zukünftig nicht. 